\title{
The pathway of molecular methods from research to routine use
}

\author{
Meissner Kristian $\ddagger$ \\ ‡ Finnish Environment Institute, SYKE, Jyväskylä, Finland
}

Corresponding author: Meissner Kristian (kristian.meissner@syke.fi)

Received: 05 Mar 2021 | Published: 08 Mar 2021

Citation: Kristian M (2021) The pathway of molecular methods from research to routine use. ARPHA Conference Abstracts 4: e65556. https://doi.org/10.3897/aca.4.e65556

\begin{abstract}
In this talk I explore how to advance molecular methods from tools in the research domain to routine use in national biomonitoring. I outline the necessity of common guidance, networks, international pilot studies and cooperation with officials to achieve the goal of method uptake into routine use. Lastly, I will explain the role that international method standardization plays in speeding up the uptake process of molecular methods into routine biomonitoring.
\end{abstract}

\section{Keywords}

molecular methods, pilot studies, validation, standardization, CEN, ISO, biomonitoring

\section{Presenting author}

Kristian Meissner, Finnish Environment Institute, SYKE

\section{Presented at}

1st DNAQUA International Conference (March 9-11, 2021) 